\title{
COMPORTAMIENTO AGRONÓMICO DE DOS SISTEMAS DE ANDROESTERILIDAD EN SORGO (Sorghum bicolor L. Moench) ${ }^{1}$
}

\author{
Víctor Pecina ${ }^{2}$ Enrique Navarro ${ }^{3}$, Héctor Williams ${ }^{4}$, Raúl Rodríguez $^{4}$
}

\begin{abstract}
RESUMEN
Comportamiento agronómico de dos sistemas de androesterilidad en sorgo (Sorghum bicolor L. Moench). La reducida variabilidad genética del sorgo y la utilización de una sola fuente de androesterilidad (Milo-Kafir, Citoplasma A1) en la producción de semilla híbrida, hacen a esta especie bastante vulnerable a posibles epifítias (plagas o enfermedades), por lo que se ha pretendido incrementar el número de fuentes de esterilidad masculina como la indicada en 1977 a la que se le denominó Citoplasma A2. Este sistema fue introducido en 1982 al Campo Experimental de Río Bravo, Tamaulipas. México; para estudiar su comportamiento en las líneas élite del programa de sorgo. El presente trabajo tuvo como objetivos: a) comparar el comportamiento agronómico de dos sistemas de androesterilidad (Citoplasma A 1 y A2), b) determinar si existen diferencias en la restauración de la fertilidad en los híbridos isocitoplásmicos. El diseño experimental fue un látice simple 7 x 7 y cuatro repeticiones. Los resultados indicaron que no existen diferencias entre los dos sistemas de androesterilidad (A1 y A2) en las características de longitud de panoja, altura de planta y rendimiento de grano, mientras que en días a floración el Citoplasma A2 fue en promedio un día más tardío que el A 1. En cuanto a la restauración de la fertilidad en los híbridos la línea $\mathrm{R}$ LRB-63 se vio afectada en los dos citoplasmas restaurando solo en forma parcial la fertilidad masculina, mientras que el CS3541 presentó una respuesta similar con las líneas LRB-1 02A, LRB-104A y LRB-110A bajo el Citoplasma A2.
\end{abstract}

\begin{abstract}
Agronomic behavior of two male sterility systems in sorghum (Sorghum bicolor L. Moench). The limited genetic variability of sorghum and the use of only one type of malesterility system (Milo-Kafir, cytoplasm A1) for the production hybrid seed, make this crop susceptible to diseases, thus its importance to look for new male sterility sources, as the cytoplasm A2 reported in 1977. This systems was introduced to the elite lines of the sorghum program at the Rio Bravo Experiment Station (INIFAP-CIRNE), in Tamaulipas, Mexico. The objective of this assay was to: a) compare the agronomic traits of two male-sterility systems (A1 and A2 cytoplasms), and b) determine if there are differences of the fertility restoration in the isocytoplasmic hybrids. The experimental design was a $7 \mathrm{x}$ 7 lattice with four replications. The results indicate that there are no differences among the two male-sterility systems (Al and A2 cytoplasms) in grain yield, plant height and panicIe length; whereas in days to blooming. the A2 cytoplasm was a day late than the Al cytoplasm. Different restoration responses were found in the hybrids, as the R LRB-63 line which partially restored fertility in the two types of cytoplasms, while CS-3541 showed a similar response with the LRB-102A, LRB-104A and LRB- $110 \mathrm{~A}$ lines in the A2 cytoplasm.
\end{abstract}

\section{INTRODUCCION}

El mejoramiento genético de plantas ha incrementado la producción y calidad de los productos vegetales, a la vez que ha provocado uniformidad en los cultivos y disminución de la variabilidad genética de éstos, por lo que al sembrar una sola variedad o diferentes variedades relacionadas entre sí, se corren grandes riesgos en la

\footnotetext{
1 Presentado en la XL Reunión Anual del PCCMCA en Costa Rica, América Central. 13 al 19 de marzo, 1994.

2 Estudiante de la Maestría en Tecnología de Semillas Actualmente Investigador de la SARH-INIFAP-CIRNE Ebano, S.L.P., México. C.P. 79150. Apdo. Postal 87.

3 Prrofesor Investigador del Departamento de Fitomejoramiento de la UAAAN. Saltillo, Coahuila, México.

4 Investigadores del Programa de Sorgo de SARH-INIF AP-CIRNE Río Bravo, Tamaulipas, México. c.P. 88900. Apdo. Postal 172.
} 
producción, debido a la reducida base genética del cultivo, ya que pueden presentarse plagas o enfermedades con resultados desastrosos.

La historia de la humanidad registra ejemplos drásticos de problemas con enfermedades, como lo ocurrido en Irlanda en 1845-46 cuando el tizón tardío (Phythophtora infestans) acabó con casi la totalidad de las áreas cultivadas con papa; en épocas más recientes han aumentado los problemas con las royas en trigo y café, así como con el hongo Helminthosporium maydis raza " $\mathrm{t}$ " en maíz, el cual en 1970 (Agrios, 1989) redujo la producción en los Estados Unidos en un 50 por ciento debido a que alrededor del 80 por ciento de la superficie cultivada con este grano poseía solamente una fuente de androesterilidad, la cual estaba asociada con la susceptibilidad a dicha enfermedad.

Como se puede observar la falta de estabilidad en la producción agrícola ha causado severos problemas en el pasado, en su mayor parte, debido a la reducida base genética de los cultivos, lo cual en cierto momento los puede hacer vulnerables a factores ambientales, por lo que deben darse acciones tendientes a reducir la vulnerabilidad, tanto en el cultivo del sorgo como en las demás especies cultivadas (Schertz y Pring, 1981).

En la actualidad la mayoría de las especies de importancia económica son genéticamente muy uniformes, como es el caso del cultivo del sorgo a nivel mundial, dado que la mayor parte de los progenitores hembra estan emparentados y los híbridos generalmente tienen los mismos progenitores masculinos; si a ello se agrega que casi en su totalidad las líneas androestériles para formar híbridos provienen originalmente de la cruza de Milo x Kafir, se incrementa el riesgo de que en un determinado momento se presente un problema fitopatológico que afecte la mayor parte del área cultivada con esta especie.

Debido a lo anterior se han buscado nuevas fuentes de androesterilidad génico-citoplásmica, las cuales sean diferentes al sistema Milo-Kafir (Citoplasma Al), encontrándose en 1976 dentro del gmpo Caudatum nigricans (raza Guinea) de Etiopía una nueva fuente a la cual se le identificó como Citoplasma A2 (Schertz, 1977).
En el año de 1982 se introdujeron al Campo Experimental de Río Bravo, Tamaulipas, México, la fuente de androesterilidad génico-citoplásmica A2, para estudiar su comportamiento en las líneas élite del programa de sorgo; Williams en 1987 reporta que es posible formar algunos híbridos con ambas fuentes (A1 y A2), ya que sus progenitores muestran la misma respuesta en los dos sistemas; además de detectar líneas de tipo R en el sistema A1 que son B en el A2, lo que aumenta la versatilidad en la formación de híbridos, al poder realizar nuevas combinaciones que no eran posibles cuando sólo se contaba con una sola fuente.

Por su parte Chisi y Miller (1987) reportan al comparar dos gmpos de híbridos unos citoplasma Al y otros con A2 (no isocitoplásmicos), que existen diferencias significativas entre éstos, en las características de rendimiento de grano, días a floración y altura de planta, no así en el grado de heterosis.

En otro estudio Rodríguez et al (1991) al estudiar un gmpo de híbridos isocitoplásmicos (A1 y A2) menciona que no existen diferencias estadísticas entre los dos sistemas de androesterilidad en las características de altura de planta, longitud de panoja, excersión, días a floración y rendimiento de grano, por lo que concluyen que el citoplasma A2 presenta un potencial de uso similar al del sistema A 1 .

Basados en lo anterior se planteó el presente trabajo con los siguientes objetivos:

a) Comparar en cuanto al comportamiento agronómico los sistemas de androesterilidad Al y A2.

b) Determinar si existen diferencias en la restauración de la fertilidad en los híbridos al cambiar el tipo de citoplasma (A1 y A2).

\section{MATERIALES Y MÉTODOS}

El presente estudio se llevó a cabo en el Campo Experimental de Río Bravo, Tamaulipas, México (INIFAPCIRNE), durante el ciclo agrícola otoño-invierno de 1989-90. 
El material genético utilizado en sus versiones A 1 y A2 de androesterilidad génico-citoplásmica fueron: las líneas LRB-102A, LRB-104A, LRB-106A y LRB-110A, mientras que los restauradores fueron: el LRB-25, LRB63, SCO-599-11E, 74CS-5388 y CS-3541; todas las líneas hembra (A) y los dos primeros restauradores (LRB) son genotipos mexicanos, obtenidos por el programa de Mejoramiento Genético de Sorgo del Campo Experimental de Río Bravo, en el estado de Tamaulipas, México (INIFAP-CIRNE); las demás líneas R fueron introducidas de Texas, E.U.A. El sistema de androesterilidad A2 se introdujo a las líneas A 1 en Río Bravo, Tam., contándose al inicio de este estudio con ocho retrocruzas hacia A2. Durante el ciclo primavera-verano de 1989 se formaron los 20 híbridos posibles y se incrementaron las cuatro líneas androestériles, ambos en sus versiones Al y A2; la semilla cosechada se utilizó para realizar las evaluaciones del presente trabajo.

Se evaluaron un total de 49 tratamientos que resultaron al combinar 20 híbridos y cuatro líneas A, con los dos sistemas de androesterilidad (citoplasma Al y A2) y un testigo comercial (RB-3030).
El diseño experimental que se utilizó fue un látice simple $7 \times 7$ con cuatro repeticiones, siendo la unidad experimental de dos surcos de $5 \mathrm{~m}$ de largo por $0,8 \mathrm{~m}$ de ancho y 20 plantas por metro lineal lo cual da una densidad de 250,000 plts/ha, el trabajo se manejó bajo condiciones de riego.

Las características evaluadas fueron: días a floración (DF), longitud de panoja (LP), altura de planta (AP), rendimiento de grano y la respuesta a la restauración de la fertilidad en los híbridos.

\section{RESULTADOS}

El análisis de los resultados indicaron diferencias altamente significativas en todas las variables evaluadas por lo cual se hizo una partición de los efectos utilizando contrastes ortogonales (Cuadro 1) pudiéndose observar en la comparación de medias que el comportamiento medio de los genotipos es superior al testigo en longitud de panoja, altura de planta y rendimiento de grano (Cuadro 2). En cuanto a la comparación de las líneas versus híbridos, éstas presentan valores inferiores a las de los híbridos como puede observarse en el Cuadro 3 , por otra parte al

Cuadro 1. Cuadrados medios del análisis de varianza de cuatro características agronómicas de sorgo para particionar el efecto de tratamientos.

\begin{tabular}{lrcllc}
\hline FV & GL & DF & LP (cm) & AP (m) & Rendimiento (kg/ha) \\
\hline Trat & 48 & $50,87^{* *}$ & $0,0057^{* *}$ & $0,0766^{* *}$ & $3,4758^{* *}$ \\
T vs (L y H) & 1 & $60,45^{*}$ & $0,0051^{*}$ & $0,0784^{* *}$ & $0,7003^{*}$ \\
Genotipos & 23 & $74,27^{* *}$ & $0,0097^{* *}$ & $0,1455^{* *}$ & $6,8777^{* *}$ \\
L vs H & 1 & $889,48^{* *}$ & $0,0133^{* *}$ & $0,8413^{* *}$ & $112,833 * *$ \\
Líneas & 3 & $6,03 \mathrm{~ns}$ & $0,0049 * *$ & $0,0735^{* *}$ & $1,0456^{* *}$ \\
Híbridos & 19 & $42,14^{* *}$ & $0,0103 * *$ & $0,1202^{* *}$ & $2,2112^{* *}$ \\
Citoplasma & 1 & $58,50^{* *}$ & $0,0030 \mathrm{~ns}$ & $0,0040 \mathrm{~ns}$ & $0,0330 \mathrm{~ns}$ \\
Genot x Cito & 23 & $11,64 \mathrm{~ns}$ & $0,0015 \mathrm{~ns}$ & $0,0107 * *$ & $0,3442 * *$ \\
(L vs H)x C & 1 & $64,23 *$ & $0,0004 \mathrm{~ns}$ & $0,0014 \mathrm{~ns}$ & $0,4712 \mathrm{~ns}$ \\
L x C & 3 & $7,53 \mathrm{~ns}$ & $0,0070 * *$ & $0,0108 *$ & $0,3896 \mathrm{~ns}$ \\
H x C & 19 & $9,52 \mathrm{~ns}$ & $0,0008 \mathrm{~ns}$ & $0,0112^{* *}$ & $0,3303 *$ \\
Error & 144 & 14,83 & 0,0012 & 0,0028 & 0,1585 \\
\hline CV $(\%)$ & & 4,7 & 11,35 & 3,49 & 8,5 \\
\hline
\end{tabular}

*,*, significativo y altamente significativo al 0,05 y $0,01 \%$ de probabilidad respectivamente. 
Cuadro 2. Comparación de medias de cuatro características agronómicas del testigo vs líneas y híbridos.

\begin{tabular}{lcccc}
\hline Genotipo & $\begin{array}{c}\text { Días a } \\
\text { Flor }\end{array}$ & $\begin{array}{c}\text { Longitud de } \\
\text { Panoja }(\mathbf{c m})\end{array}$ & $\begin{array}{c}\text { Altura de } \\
\text { Planta }(\mathbf{m})\end{array}$ & $\begin{array}{c}\text { Rendto } \\
(\mathbf{k g} / \mathbf{h a})\end{array}$ \\
\hline RB-3030 $(\mathrm{T})$ & $78 \mathrm{a}$ & $26,7 \mathrm{~b}$ & $1,39 \mathrm{~b}$ & $5,287 \mathrm{~b}$ \\
$\mathrm{~L} \mathrm{y} \mathrm{H}$ & $82 \mathrm{~b}$ & $30,7 \mathrm{a}$ & $1,53 \mathrm{a}$ & $5,764 \mathrm{a}$ \\
\hline
\end{tabular}

Valores en la misma columna con distinta letra son estadísticamente diferentes (Duncan a 0,05).

Cuadro 3. Medias de cuatro características agronómicas de sorgo promedio de líneas vs híbridos.

\begin{tabular}{lcccc}
\hline Genotipo & $\begin{array}{c}\text { Días a } \\
\text { Flor }\end{array}$ & $\begin{array}{c}\text { Longitud de } \\
\text { Panoja }(\mathbf{c m})\end{array}$ & $\begin{array}{c}\text { Altura de } \\
\text { Planta }(\mathbf{m})\end{array}$ & $\begin{array}{c}\text { Rendto } \\
(\mathbf{k g} / \mathbf{h a})\end{array}$ \\
\hline Híbridos & $81 \mathrm{a}$ & $30,7 \mathrm{a}$ & $1,56 \mathrm{a}$ & $6,178 \mathrm{a}$ \\
Líneas & $87 \mathrm{~b}$ & $28,5 \mathrm{~b}$ & $1,38 \mathrm{~b}$ & $3,695 \mathrm{~b}$ \\
\hline
\end{tabular}

Valores en la misma columna con distinta letra son estadísticamente diferentes (Duncan a 0,05).

realizar una comparación entre líneas puede detectarse que éstas difieren sólo en las características de longitud de panoja, altura de planta y rendimiento de semilla siendo las mejores la LRB-104A y LRB-106A (Cuadro 4). En cuanto a los híbridos se detectó que los más productivos provienen de la cruza de las cuatro líneas hembra con el restaurador LRB-25 (Cuadro 5), además se observóque existe correlación entre la longitud de panoja y el rendimiento en los cinco mejores híbridos, sin embargo son más tardíos y altos.

Con respecto a la comparación de los dos sistemas de androesterilidad (Citoplasma Al y A2) sólo se detectó diferencias estadísticas en días a floración (Cuadro 6) resultando los genotipos con citoplasma A2 más tardíos en promedio de un día, en las demás características son estadísticamente iguales.

En lo referente a la restauración de la fertilidad masculina en los híbridos, pudo detectarse que existen diferencias entre genotipos y en la interacción genotipo por citoplasma, observándose en el Cuadro 7 que los genotipos provenientes de la cruza con los restauradores LRB25 , SCO-599-11E y 74CS-5388 presentan óptimos porcentajes de grano bajo los dos sistemas de androesterilidad ( $\mathrm{Al}$ y A2), por lo que se asume que hubo una efectiva restauración de la fertilidad masculina pues fueron aisladas cinco panojas de cada genotipo antes de la floración para detectar esta variable; también se observó que el LRB-63 se ve afectado bajo las dos condiciones en tres de las líneas LRB-102, LRB-104 y LRB-106, mientras que en la LRB-110 sólo modifica su comportamiento en el citoplasma A2, por otra parte el CS-3541 presenta una respuesta diferente en el citoplasma A2 en las líneas LRB-102, LRB-104 y LRB-106.

En cuanto a los coeficientes de variación, el más alto lo presenta la característica de longitud de panoja con 11,35 por ciento, en los demás caracteres los valores son más bajos (Cuadro 1).

\section{DISCUSIÓN}

Los análisis de varianza y la comparación de medias (Cuadros 1 y 2) detectaron diferencias significativas entre el testigo y los demás genotipos $(\mathrm{L}$ y $\mathrm{H}$ ) para las cuatro características evaluadas (días a floración, longitud de panoja, altura de planta y rendimiento), pudiéndose observar que existen híbridos que son superiores en la mayoría de los caracteres al testigo aunque son un poco más 
Cuadro 4. Medias de cuatro características agronómicas de sorgo de cuatro líneas androestériles.

\begin{tabular}{lcccc}
\hline Genotipo & $\begin{array}{c}\text { Días a } \\
\text { Flor }\end{array}$ & $\begin{array}{c}\text { Longitud de } \\
\text { Panoja }(\mathbf{c m})\end{array}$ & $\begin{array}{c}\text { Altura de } \\
\text { Planta }(\mathbf{m})\end{array}$ & $\begin{array}{c}\text { Rendto } \\
(\mathbf{k g} / \mathbf{h a})\end{array}$ \\
\hline LRB-102 & $87 \mathrm{a}$ & $27,6 \mathrm{~b}$ & $1,49 \mathrm{a}$ & $3,373 \mathrm{~b}$ \\
LRB-104 & $86 \mathrm{a}$ & $31,8 \mathrm{a}$ & $1,28 \mathrm{~b}$ & $3,955 \mathrm{a}$ \\
LRB-106 & $87 \mathrm{a}$ & $26,0 \mathrm{~b}$ & $1,44 \mathrm{a}$ & $4,176 \mathrm{a}$ \\
LRB-110 & $88 \mathrm{a}$ & $28,5 \mathrm{ab}$ & $1,33 \mathrm{~b}$ & $3,279 \mathrm{~b}$ \\
\hline
\end{tabular}

Valores en la misma columna con distinta letra son estadísticamente diferentes (Duncan a 0,05).

Cuadro 5. Medias de cuatro características agronómicas de los mejores cinco híbridos de sorgo.

\begin{tabular}{lcccc}
\hline Genotipo & $\begin{array}{c}\text { Días a } \\
\text { Flor }\end{array}$ & $\begin{array}{c}\text { Longitud de } \\
\text { Panoja }(\mathbf{c m})\end{array}$ & $\begin{array}{c}\text { Altura de } \\
\text { Planta }(\mathbf{m})\end{array}$ & $\begin{array}{c}\text { Rendto } \\
(\mathbf{k g} / \mathbf{h a})\end{array}$ \\
\hline LRB-102 x LRB-25 & $83 \mathrm{~d}$ & $35,5 \mathrm{a}$ & $1,69 \mathrm{e}$ & $7,498 \mathrm{a}$ \\
LRB-104 x LRB-25 & $85 \mathrm{~d}$ & $35,0 \mathrm{a}$ & $1,53 \mathrm{c}$ & $7,373 \mathrm{a}$ \\
LRB-106 x LRB-25 & $84 \mathrm{~d}$ & $33,7 \mathrm{a}$ & $1,60 \mathrm{~d}$ & $7,225 \mathrm{a}$ \\
LRB-110 x LRB-25 & $83 \mathrm{~d}$ & $33,2 \mathrm{a}$ & $1,63 \mathrm{~d}$ & $6,848 \mathrm{~b}$ \\
LRB-104 x CS-3541 & $81 \mathrm{~d}$ & $29,8 \mathrm{~b}$ & $1,68 \mathrm{e}$ & $6,654 \mathrm{~b}$ \\
\hline
\end{tabular}

Valores en la misma columna con distinta letra son estadísticamente diferentes (Duncan a 0,05).

Cuadro 6. Medias de cuatro características agronómicas de sorgo de acuerdo con el sistema de androesterilidad.

\begin{tabular}{lcccc}
\hline $\begin{array}{l}\text { Fuente de } \\
\text { Androesterilidad }\end{array}$ & $\begin{array}{c}\text { Días a } \\
\text { Flor }\end{array}$ & $\begin{array}{c}\text { Longitud de } \\
\text { Panoja }(\mathbf{c m})\end{array}$ & $\begin{array}{c}\text { Altura de } \\
\text { Planta (m) }\end{array}$ & $\begin{array}{c}\text { Rendto } \\
(\mathbf{k g} / \mathbf{h})\end{array}$ \\
\hline Citoplasma A1 & $81 \mathrm{a}$ & $28,7 \mathrm{a}$ & $1,53 \mathrm{a}$ & $4,667 \mathrm{a}$ \\
Citoplasma A2 & $82 \mathrm{~b}$ & $28,5 \mathrm{a}$ & $1,54 \mathrm{a}$ & $4,640 \mathrm{a}$ \\
\hline
\end{tabular}

Valores en la misma columna con distinta letra son diferentes estadísticamente al $0,05 \%$ de probabilidad (Duncan).

Cuadro 7. Restauración de la fertilidad en los 20 híbridos de sorgo bajo dos sistemas de androesterilidad.

\begin{tabular}{|c|c|c|c|c|c|}
\hline \multirow[t]{2}{*}{ Genotipo } & \multicolumn{2}{|c|}{ Citoplasma } & \multirow[t]{2}{*}{ Genotipo } & \multicolumn{2}{|c|}{ Citoplasma } \\
\hline & A1 & $\overline{\mathrm{A} 2}$ & & $\overline{\mathrm{A1}}$ & $\overline{\mathrm{A2}}$ \\
\hline LRB-102 x LRB-25 & 1 & 1 & LRB-106 x LRB-25 & 1 & 1 \\
\hline LRB-102 x LRB-63 & 3 & 4 & LRB-106 x LRB-63 & 3 & 4 \\
\hline LRB-102 x CS-3541 & 1 & 4 & LRB-106 x CS-3541 & 1 & 4 \\
\hline LRB-102 x SCO-599-11E & 1 & 1 & LRB-106 x SCO-599-11E & 1 & 1 \\
\hline LRB-102 x 74CS-5388 & 1 & 1 & LRB-106 x 74CS-5388 & 1 & 1 \\
\hline LRB-104 x LRB-25 & 1 & 1 & LRB-110 x LRB-25 & 1 & 1 \\
\hline LRB-104 x IRB-63 & 4 & 4 & LRB-110 x LRB-63 & 1 & 3 \\
\hline LRB-104 x CS-3541 & 1 & 4 & LRB-110 x CS-3541 & 1 & 1 \\
\hline LRB-104 x SCO-599-11E & 1 & 1 & LRB-110 x SCO-599-11 & 1 & 1 \\
\hline LRB-104 x 74CS-5388 & 1 & 1 & LRB-110 x 74CS-5388 & 1 & 1 \\
\hline
\end{tabular}

Porcentaje de grano 1) 100-76, 2) $75-51,3) 50-26,4) 25-1,5) 0$. 
tardíos y altos, por lo que en un futuro dependiendo de su comportamiento bien pudieran substituir a éste ventajosamente. En cuanto a la comparación de medias líneas vs híbridos (Cuadro 3) se observó que los híbridos superan a todas las líneas, lo cual debe ser normal si se considera que se está explotando el vigor híbrido y las líneas son materiales autofecundados de ahí que se presente tal comportamiento.

Con respecto al comportamiento medio de las líneas (Cuadro 4) la comparación de medias permite detectar las mejores líneas en cuanto a características agronómicas, sin embargo esto no es garantía de que tal comportamiento les sea heredado a la progenie híbrida ya que dependerá del grado aptitud combinatoria (general o específica) que exhiba con los diferentes restauradores, como puede observarse en la comparación de los diferentes híbridos (Cuadro 5); también se detectó que aún cuando los mejores híbridos en rendimiento tanto en el citoplasma A1 y A2 presentan el mismo restaurador (LRB-25) es obvio que existe un diferente nivel de heterosis entre éstos, no así entre los dos sistemas de androesterilidad lo cual coincide con lo reportado por Chisi y Miller (1987).

En cuanto a los dos sistemas de androesterilidad (citoplasma A1 y A2) el análisis de varianza detectaron diferencias estadísticas sólo en días a floración, sin embargo tal diferencia se reduce únicamente a un día en promedio (Cuadro 6) lo cual no es significativo en la práctica y al no encontrar diferencias en las demás características se ratifica lo encontrado por Rodríguez et al. (1991) al estudiar estos mismos genotipos.

Por otra parte al analizar la interacción híbrido-citoplasma se detectó que algunos híbridos presentan diferente respuesta bajo uno u otro citoplasma en altura de planta y rendimiento, mientras que otros no modifican su comportamiento, lo cual es importante, ya que puede producirse semilla de un mismo híbrido en dos versiones (A1 y A2), de tal forma que se podría mezclar la semilla en forma proporcional, disminuyendo así la vulnerabilidad genética del cultivo al aumentar la diversidad en los citoplasmas.
En cuanto a la restauración de la fertilidad masculina (Cuadro 7) se detectó que los restauradores LRB-63 y CS-3541 presentan una interacción con el citoplasma, el primero en A 1 y A2 y el segundo sólo bajo el citoplasma A2 en tres de las líneas (LRB-102, LRB-104 y LRB-110) pudiendo haber una restauración parcial o total de la fertilidad dependiendo del genotipo.

En cuanto a los coeficientes de variación el más alto lo presenta la longitud de panoja con 11,35 por ciento considerándose aceptable y por lo tanto puede asumirse que los resultados tienen un alto grado de confiabilidad.

\section{CONCLUSIONES}

Con base en los resultados obtenidos y bajo las condiciones en que se condujo el presente estudio se puede concluir que:

1. No se detectaron diferencias entre los dos sistemas de androesterilidad (citoplasma $\mathrm{Al}$ y A2) en las características de longitud de panoja, altura de planta y rendimiento de grano.

2. En días a floración se observaron diferencias estadísticas entre los dos tipos de citoplasmas, resultando los materiales con citoplasma A2 más tardíos en un día en promedio.

3. En la restauración de la fertilidad masculina en los híbridos, se detectó que la línea LRB-63 interaccionó con los dos citoplasmas, restaurando en forma parcial la fertilidad. El CS-3541 cambió su respuesta en el citoplasma A2, en tres de las líneas.

\section{LITERATURA CITADA}

AGRIOS, N. G. 1989. Fitopatología. Traducción de Guzmán, O. M. Primera Edición. México. D.F. Editorial Limusa. 756 p.

CHISI, M.; MILLER, R. 1987. Comparision of F1 hybrids in A1 and A2 cytoplasm across locations. Sorghum Newsletter. 30:23-24. 
RODRIGUEZ, H. R; WILIAMS, A. H.; TORRES, M. H. 1991. Comparación agronómica de dos tipos de esterilidad génico-citoplásmica en sorgo. Campo Experimental Río Bravo. SARH. INIFAP. In: Memorias del II Congreso Nacional de Genética. Saltillo, Coahuila, México. p. 19.

SCHERTZ, K. F. 1977. Registration of A2 Tx2753 and B Tx2753, sorghum germoplasm. Crop Science. 17:983.
; PRING, D. R. 1981. Cytoplasmic sterility systems in sorghum. In: House, L.R, L.R Mughogho and J.M. Peacock. (eds). Sorghum in the Eighties. ICRISAT. Patancheru, A.P. India. pp: 373-384.

WILLIAMS, H. A. 1987. Respuesta de líneas experimentales y comerciales de sorgo al sistema de androesterilidad génico-citoplásmica A2. Fitotecnia. México. 10:91-98. 